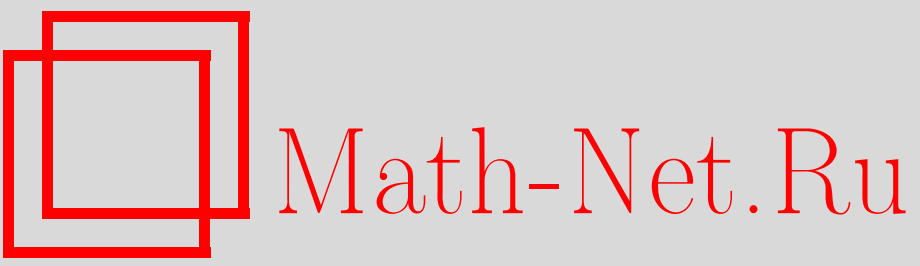

В. Д. Николаев, Атаки на схемы электронной подписи, не учитываемые традиционными определениями стойкости, и меры противодействия им, Матем. вопр. криптогр., 2016, том 7, выпуск 1, 93-118

DOI: https://doi.org/10.4213/mvk177

Использование Общероссийского математического портала Math-Net.Ru подразумевает, что вы прочитали и согласны с пользовательским соглашением

http://www.mathnet.ru/rus/agreement

Параметры загрузки:

IP : 54.196.121.252

26 апреля 2023 г., 07:51:24 
МАТЕМАТИЧЕСКИЕ ВОПРОСЫ КРИПТОГРАФИИ

2016 T. 7 № 1 C. 93-118

УДК 519.719.2

\title{
Атаки на схемы электронной подписи, не учитываемые традиционными определениями стойкости, и меры противодействия им
}

\author{
В. Д. Николаев
}

ООО «КРИПТО-ПРО», Москва

Получено 8.IX.2015

Изучается возможность проведения атак дублирования подписи на выбранном ключе на различные схемы электронной подписи. Рассматриваются меры противодействия таким атакам как на уровне протоколов, так и на уровне схем подписи.

Работа выполнена при финансовой поддержке Академии криптографии Российской Федерации.

Ключевые слова: электронная подпись, DSKS, криптографические протоколы, доказуемая стойкость

Attacks on digital signature schemes not accounted by the common security definitions, and countermeasures

\section{D. Nikolaev}

CryptoPro, LLC, Moscow

Abstract. The implementation of the so called duplicate signature key selection attack on different digital signature schemes is discussed. We discuss also the usage of this attack for breaking some cryptographic protocols based on vulnerable signature schemes. Countermeasures for preventing this attack at the scheme-level and protocol-level are considered as well.

Keywords: digital signature, DSKS, cryptographic protocols, provable security 


\section{1. Введение}

Для установления связи между документом и создавшим его лицом давно используется такой инструмент, как подпись. Долгое время был известен только один тип подписи - собственноручная подпись. С развитием электронновычислительных устройств возникла необходимость создания новых механизмов подписи, которые позволяли бы ускорить и автоматизировать процессы подписания и проверки подписи. Таким механизмом стала электронная подпись (ЭП).

Введем некоторые базовые определения (см., например, [19]).

Определение 1. Схема электронной подписи есть тройка пар алгоритмов $\left(D_{G}, D_{V}\right),\left(G_{G}, G_{V}\right),\left(\Sigma_{G}, \Sigma_{V}\right)$, где:

- $D_{G}$ - алгоритм выработки доменных параметров - рандомизированный алгоритм, который принимает на вход некоторый параметр безопасности и возвращает набор доменных параметров $D$ и, возможно, дополнительную информацию $I$, позволяющую проверить, что доменные параметры удовлетворяют определенным требованиям безопасности;

- $D_{V}$ - алгоритм проверки корректности доменных параметров - детерминированный алгоритм, принимающий на вход набор доменных параметров $D$, а также, возможно, дополнительную информацию $I$ и возвращающий один бит, описывающий соответствие доменных параметров требованиям безопасности;

- $G_{G}$ - алгоритм выработки ключевой пары - рандомизированный алгоритм, принимающий на вход набор доменных параметров $D$ и возвращающий ключевую пару (priv, pub), где priv - секретный ключ, а pub - соответствующий открытый ключ;

- $G_{V}$ - алгоритм проверки обладания секретным ключом - алгоритм, выполняемый двумя участниками - доказывающим и проверяющим. Оба участника обладают парой $(D, p u b)$, где $D$ - набор доменных параметров, a pub - открытый ключ; доказывающий также обладает секретным ключом priv, соответствующим открытому ключу pub; в ходе выполнения алгоритма доказывающий должен убедить проверяющего в обладании секретным ключом;

- $\Sigma_{G}$ - алгоритм выработки подписи (алгоритм подписания) - рандомизированный алгоритм, принимающий на вход сообщение $m$, секретный ключ priv и набор доменных параметров $D$ и возвращающий значение подписи $s$; 
- $\Sigma_{V}$ - алгоритм проверки подписи (алгоритм верификации) - детерминированный алгоритм, принимающий на вход сообщение $m$, значение подписи $s$, набор доменных параметров $D$, открытый ключ pub и возвращающий сообщение «Принять» или «Отвергнуть».

Использование схем ЭП должно обеспечивать выполнение следующих условий (см., например, [15]):

- аутентификация участника,

- аутентификация источника данных,

- невозможность отказа от авторства,

- контроль целостности данных.

Эти условия позволяют использовать данный примитив в различных протоколах, в том числе и для проведения аутентификации. Одним из таких протоколов является протокол выработки общего ключа STS (station-to-station, [8]). Протокол позволяет двум его участникам выработать общий секретный ключ; при этом сложность получения секретного ключа противником, просматривающим канал передачи сообщений, эквивалентна сложности решения вычислительной задачи Диффи - Хеллмана.

Определение 2. Вычислительная задача Диффи-Хеллмана (computational Diffie-Hellman problem) есть задача нахождения в циклической группе $G$ с образующим элементом $g$ по данным $g^{x}, g^{y}$ элемента $g^{x y} .{ }^{1)}$

В протоколе STS схема ЭП используется для взаимной аутентификации участников. Для этого каждый участник подписывает передаваемое сообщение и вместе с ним отправляет сертификат открытого ключа проверки подписи. Получатель проверяет подпись при помощи открытого ключа и проверяет подлинность сертификата, используя так называемую инфраструктуру открытых ключей (PKI - public key infrastructure), которая позволяет доверенным образом связать сертификат с владельцем - физическим лицом, организацией или сервисом. Если проверка проходит успешно, то личность второго участника считается удостоверенной.

Однако в [8] и [5] были указаны некоторые сценарии атак на протокол STS, в том числе сценарий атаки Uknown Key Share (UKS), другое название «source-substitution attack» (атака с подменой источника).

\footnotetext{
1) Существуют группы, для которых не найдено эффективных алгоритмов решения вычислительной задачи Диффи - Хеллмана.
} 
Определение 3. Атака Unknown Key Share (UKS) на протокол выработки общего ключа - атака, в результате которой честный участник Алиса уверена, что общий секретный ключ выработан с другим честным участником Бобом, как это и есть на самом деле, в то время как Боб полагает, что ключ был выработан с третьим участником Мэллори.

В [5] Блейк-Уилсон и Менезес более подробно рассмотрели атаку UKS на протокол STS и ввели понятие свойства схемы ЭП - DSKS (Duplicate Signature Key Selection, вариант русского перевода - дублирование подписи на выбранном ключе), которое использовалось для атаки на протокол в целом.

Определение 4. Схема ЭП обладает свойством дублирования подписи на выбранном ключе, если противник может по известному открытому ключу проверки подписи участника $A$, некоторому сообщению $M$ и значению подписи $s_{A}$ этого сообщения, выработанной участником $A$, выбрать свою ключевую пару $\operatorname{priv}_{C}, \operatorname{pub}_{C}$ таким образом, что верификация подписи $s_{A}$ сообщения $M$ на открытом ключе проверки подписи данной пары пройдет успешно.

Более строго, у противника есть полиномиальный рандомизированный алгоритм, принимающий на вход набор доменных параметров $D$, открытый ключ $\operatorname{pub}_{A}$ участника $A$, сообщение $M$, значение подписи $s_{A}$ и возвращающий такую ключевую пару $\operatorname{priv}_{C}, \operatorname{pub}_{C}$, что $\Sigma_{V}\left(M, s_{A}, D, \operatorname{pub}_{C}\right)=$ «Принять».

В [5] также показано наличие данного свойства у некоторых схем ЭП. Необходимо отметить, что при демонстрации наличия данного свойства у схемы ЭП типа Эль-Гамаля авторы меняли некоторые параметры используемой алгебраической группы, которые входили в набор доменных параметров.

Определение 5. Будем говорить, что схема ЭП обладает свойством дублирования подписи на выбранном ключе с измененными доменными параметрами, если существует полиномиальный рандомизированный алгоритм, принимающий на вход набор доменных параметров $D$, открытый ключ рub $_{A}$ участника $A$, сообщение $M$, значение подписи $s_{A}$ и возвращающий такой новый набор доменных параметров $D^{\prime}$ и такую ключевую пару $\operatorname{priv}_{C}, \operatorname{pub}_{C}$, что $\Sigma_{V}\left(M, s_{A}, D^{\prime}, \operatorname{pub}_{C}\right)=\langle$ Принять».

В [16] Коблиц и Менезес используют при введении определений термин «атака» вместо термина «свойство»; далее мы также будем использовать термин «атака». В той же работе рассматривался и второй вариант атаки - с использованием секретного ключа подписи, что позволяло для некоторых схем ЭП проводить DSKS, не меняя при этом доменных параметров. 
В более ранней работе [19] дополнительно вводились определения «сильной» и «слабой» DSKS. «Слабая» атака предполагает нахождение только открытого ключа, «сильная» - и соответствующего секретного. Очевидно, что определение «сильной» DSKS соответствует определению 4; в дальнейшем под DSKS будем подразумевать «сильную» DSKS.

B [22] вводится несколько более широкое определение и рассматривается еще один вариант данной атаки:

Определение 6 (Rosa, [22]). Будем говорить, что кортеж

$$
\left[\operatorname{pub}_{A}, D_{A}, M_{A}, \operatorname{pub}_{B}, D_{B}, M_{B}, s\right],
$$

в котором $\operatorname{pub}_{X}$ - открытый ключ участника $X, s-$ значение подписи, $M_{X}$ - сообщение участника $X$, формирует $k$-коллизию $(k=1,2)$, если:

- $\operatorname{pub}_{A} \neq \operatorname{pub}_{B}$

- проверка $s$ как подписи сообщения $M_{A}$ на открытом ключе проверки $\operatorname{pub}_{A}$ проходит успешно (более формально, если $\Sigma_{V}\left(M_{A}, s, D_{A}, \operatorname{pub}_{A}\right)=$ $=\ll$ Принять»);

- проверка $s$ как подписи сообщения $M_{B}$ на открытом ключе проверки $\mathrm{pub}_{B}$ проходит успешно (более формально, если $\Sigma_{V}\left(M_{B}, s, D_{B}, \mathrm{pub}_{B}\right)=$ $=$ (Принять»).

Если при этом $M_{A}=M_{B}$, то будем говорить об 1-коллизии, в противном случае - о 2-коллизии.

Если $D_{A} \neq D_{B}$, то будем говорить о $k$-коллизии по Роса с измененныли доменными параметрами.

Заметим, что «слабая» атака DSKS есть нахождение 1-коллизии.

Определение 7 (Goldwasser-Micali-Rivest (GMR-стойкость) [13, 16]). Будем говорить, что схема ЭП является $(t, \varepsilon, q)$-стойкой к экзистенциальной подделке на основе атаки с адаптивным выбором сообщений, если ни один противник, который может получить подписи не более $q$ выбранных им сообщений, не сможет подделать подпись любого другого сообщения за время, меньшее $t$, с вероятностью, большей $\varepsilon$.

В данной работе ставится вопрос об уязвимости схем ЭП к атаке, которая, по утверждению авторов [16], не нарушает условия, указанные в широко используемом определении GMR-стойкости схемы ЭП, но, тем не менее, может в некоторых ситуациях угрожать безопасности систем, использующих данные схемы ЭП. 


\section{2. Возможные применения атаки DSKS}

Предполагается, что подпись (электронная или собственноручная) дает проверяющему уверенность, что никто другой не сможет объявить подпись своей, а настоящий подписавший не сможет отказаться от авторства. Схемы подписи, которые уязвимы к различным разновидностям атаки DSKS, в некоторых условиях этими свойствами не обладают. Рассмотрим ряд ситуаций.

\section{1. Анонимный конкурс}

Коблиц и Менезес в статье [16] описали следующую ситуацию. Предположим, что проводится анонимный конкурс, победитель которого получает ценный приз. Работы подписываются авторами и отправляются на проверку. Победитель доказывает свое авторство, предоставляя открытый ключ для верификации подписи. Если происходит утечка данных от жюри конкурса, то противник может сгенерировать собственную ключевую пару так, что подпись работы-фаворита на этом ключе пройдет проверку. Тогда сразу после объявления итогов противник предъявляет эту ключевую пару, забирает приз и скрывается с ним до появления настоящего автора.

\section{2. Подписание контракта}

Следующая ситуация также описана в [16]. Предположим, Алиса и Крис заключают между собой контракт, который оба подписывают при помощи схемы ЭП, уязвимой к атаке DSKS. Крис, не желающий выполнять условия контракта, заранее создает несколько ключевых пар, которые удовлетворяют DSKS-свойству, и получает сертификаты этих ключевых пар, оформленные на подставных лиц. Когда Алиса подает на него в суд, Крис предъявляет сертификаты открытых ключей этих пар, обладатели которых также могли подписать этот договор.

\section{3. Протокол выработки общего ключа STS}

Схемы электронной подписи часто используются в качестве примитивов в различных криптографических протоколах. Как говорилось выше, уязвимость примитива к атаке DSKS может привести к уязвимости всего протокола к некоторым атакам.

Одним из важных свойств, которым должен обладать протокол выработки общего ключа, является его стойкость к атаке типа unknown key share. Предполагается, что противник не может заставить одну из сторон поверить, что общий ключ вырабатывается с ним, а не с другим участником, как это есть в реальности. Рассмотрим протокол выработки общего ключа STS (вариацию STS-MAC, см., например, [5]). Сначала кратко опишем его. 
Предположим, что стороны $A$ и $B$ хотят выработать общий ключ. Заранее выбирается циклическая группа, определяется ее порядок $n$ и выбирается образующий элемент $a$; стороны также выбирают используемую схему электронной подписи и набор ее доменных параметров $D$, получая затем ключевые пары $\left(\operatorname{priv}_{A}, \operatorname{pub}_{A}\right)$ и $\left(\operatorname{priv}_{B}, \operatorname{pub}_{B}\right)$. Далее выполняются следующие действия.

1. $A$ выбирает секретный параметр $x \in_{R}\{1,2, \ldots, n-1\}$ и пересылает $B$ сообщение:

$$
A \rightarrow B: a^{x} .
$$

2. $B$, получив сообщение (1), выбирает, в свою очередь, секретный параметр $y \in{ }_{R}\{1,2, \ldots, n-1\}$, вычисляет общий ключ $K=\left(a^{x}\right)^{y}$, после чего отправляет $A$ сообщение:

$$
B \rightarrow A: a^{y}, s_{B}\left(a^{x} \| a^{y}\right), M A C_{K}\left(s_{B}\left(a^{x} \| a^{y}\right)\right), \operatorname{cert}_{B},
$$

где $s_{B}(M)=\Sigma_{G}\left(M, \operatorname{priv}_{B}, D\right)$ есть электронная подпись сообщения $M$ на секретном ключе участника $B, M A C_{K}$ - ключевая функция хэширования на ключе $K, \operatorname{cert}_{B}-$ сертификат открытого ключа подписи участника $B$.

3. $A$, получив сообщение (2), вычисляет общий ключ $K=\left(a^{y}\right)^{x}$, проверяет значение МАC полученной подписи, проверяет подпись участника $B$, после чего отправляет $B$ сообщение:

$$
A \rightarrow B: s_{A}\left(a^{x} \| a^{y}\right), M A C_{K}\left(s_{A}\left(a^{x} \| a^{y}\right)\right), \operatorname{cert}_{A},
$$

где $s_{A}(M)=\Sigma_{G}\left(M, \operatorname{priv}_{A}, D\right), \operatorname{cert}_{A}$ - сертификат открытого ключа подписи участника $A$.

4. $B$, получив сообщение (3), также проверяет значение $M A C$ полученной подписи и саму подпись участника $A$.

К сожалению, протокол оказывается уязвимым к атаке UKS, если используемая схема электронной подписи уязвима к атаке DSKS. Опишем атаку, предложенную в [5]. Предположим, что Алиса желает выработать общий ключ с Бобом, для чего инициирует протокол. Активный противник Мэллори перехватывает сообщение (1), узнаёт $a^{x}$ и отправляет то же сообщение Бобу как свое. После того как Боб ответил, Мэллори просматривает сообщение (2), узнаёт $a^{y}$ и пересылает сообщение Алисе. Когда Алиса отвечает сообщением (3), Мэллори его вновь перехватывает, определяет новую ключевую пару $\left(\operatorname{priv}_{M}, \operatorname{pub}_{M}\right)$ и, возможно, новый набор доменных параметров $D^{\prime}$ так, что $\Sigma_{V}\left(\left(a^{x}|| a^{y}\right), s_{A}\left(a^{x} \| a^{y}\right), D^{\prime}, \operatorname{pub}_{M}\right)=$ «Принять», получает сертификат открытого ключа полученной пары $\operatorname{cert}_{M}$ и подменяет им $\operatorname{cert}_{A}$. 
Таким образом, Боб, проверив подпись после получения сообщения (3), будет уверен, что выработал общий ключ с Мэллори, хотя на самом деле установил его с Алисой, т. е. свойство стойкости к атаке UKS более не выполняется.

\section{4. Протокол сертифицированной почты}

В [24] Шнайер и Риордан предложили почтовый протокол, который должен обладать следующими двумя свойствами:

- отправитель $A$ должен иметь возможность проверить, что адресат получил письмо, а в случае если последний заявит, что письмо не получил, сможет доказать обратное;

- адресат $B$ должен иметь возможность доказать, что не получил письмо от отправителя, если последний заявит обратное.

Опишем протокол. Заранее оговариваются алгоритм $E$ шифрования, хэшфункция $h$ и схема электронной подписи $\left(\left(D_{G}, D_{V}\right),\left(G_{G}, G_{V}\right),\left(\Sigma_{G}, \Sigma_{V}\right)\right)$ с набором доменных параметров $D$. Адресат вырабатывает ключевую пару $\left(\operatorname{priv}_{B}, \operatorname{pub}_{B}\right) ;$ он также должен иметь возможность представить сертификат своего открытого ключа электронной подписи, который может быть использован отправителем для проверки подписи. Затем стороны производят следующие действия:

- отправитель выбирает случайный ключ $K$ и отправляет адресату сообщение: $E_{K}($ text), где text - текст письма;

- адресат, получив шифртекст, отвечает сообщением $M$ и значением своей подписи $s_{B}(M)=\Sigma_{G}\left(M, \operatorname{priv}_{B}, D\right)$, где $M$ - сообщение следующего вида: «Я хочу, чтобы отправитель опубликовал ключ для шифртекста, хэш-значение которого равно $h\left(E_{K}(\mathrm{text})\right)$, до наступления даты date на сервере $X »$;

- отправитель публикует кортеж $\left(h\left(E_{K}(\mathrm{text})\right), K\right)$ на сервере $X$ до даты date;

- адресат получает ключ и расшифровывает шифртекст.

Свойства протокола обеспечиваются следующим образом. Если адресат вдруг заявит, что письмо не получил, то отправитель пересылает арбитру кортеж (text, $K, E_{K}$ (text), $\left.s_{B}(M), X\right)$. Арбитр проверяет правильность шифрования, хэш-значения $E_{K}($ text), факт публикации, подпись адресата. Если все проходит корректно, арбитр убеждается в правоте отправителя. Если же адресат хочет доказать арбитру, что письмо не получил, то арбитр запрашивает те же данные у отправителя, и если отправитель не может их представить, признает правоту адресата. 
К сожалению, данный протокол не обладает первым свойством, если используемая схема электронной подписи уязвима к атаке DSKS. В этом случае адресат может создать такую новую ключевую пару $\operatorname{priv}_{C}, \operatorname{pub}_{C}$, что $\Sigma_{V}\left(M, s_{B}(M), D, \operatorname{pub}_{C}\right)=$ «Принять». Затем, после завершения протокола, он заявляет, что никакого письма не получал. Отправитель пересылает данные арбитру, к которому является подставное лицо, предъявляющее сертификат открытого ключа $C$ и заявляющее, что письмо на самом деле получило именно оно.

\section{3. Атаки на различные схемы электронной подписи}

\section{1. Основные принципы}

Коблиц и Менезес в [16] описывают DSKS-атаки на схему RSA, схему Рабина и ECDSA ([10]). Описанные ими атаки осуществляются следующим образом: атакующий подменяет один или несколько доменных параметров схемы, а затем подбирает новую ключевую пару так, чтобы она удовлетворяла равенствам, используемым для проверки подписи. Рассмотрим применение этого приема для некоторых схем ЭП.

Для описания типа проводимой атаки DSKS будем использовать следующую нотацию. Будем говорить о $i$-jk-DSKS, где $i \in\{1,2\}, j \in\{d, n\}, k \in\{w, s\}$, если в результате сильной $(k=s)$ или слабой $(k=w)$ атаки DSKS с измененными $(j=d)$ или постоянными $(j=n)$ доменными параметрами была построена $i$-коллизия по Роса.

\section{2. Схема подписи RSA}

Сначала опишем схему RSA (см., например, [21]). С помощью алгоритма выработки доменных параметров заранее определяется набор доменных параметров $D$ : хэш-функция hash, битовые длины простых сомножителей. Каждый участник вырабатывает ключевую пару следующим образом: выбираются такие два простых числа $p, q$, что $p \neq q, p$ и $q$ имеют оговоренную битовую длину, вычисляется значение $N=p q$. Затем случайным образом выбирается число $e: 1<e<\varphi(N)$ и $(e, \varphi(N))=1$, где $\varphi(N)$ - значение функции Эйлера. Далее вычисляется значение $d=e^{-1} \bmod \varphi(N)$. Секретный ключ есть кортеж $(p, q, d)$, открытый - $(N, e)$. Алгоритм подписания $\Sigma_{G}$ при получении на вход кортежа $[M,(p, q, d), D]$, где $M$ - подписываемое сообщение, возвращает $s=h^{d} \bmod N$, где $h=\operatorname{hash}(M)$. Алгоритм проверки подписи $\Sigma_{V}$ на входе $[M, s, D,(N, e)]$ возвращает «Принять» тогда и только тогда, когда $s^{e}=h \bmod N$. 
Теперь опишем атаку DSKS типа 1-ns-DSKS, предложенную в [16]. Противник может выбрать такие простые $p^{\prime}, q^{\prime}$, что $N^{\prime}=p^{\prime} q^{\prime}$ имеет необходимую битовую длину, при этом $p^{\prime}-1$ и $q^{\prime}-1$ раскладываются на небольшие множители, $s$ и $h$ являются образующими элементами $F_{p^{\prime}}^{*}$ и $F_{q^{\prime}}^{*}$, а числа $\left(p^{\prime}-1\right) / 2$ и $\left(q^{\prime}-1\right) / 2$ взаимно просты. Тогда противник решает сравнения $s^{x}=h \bmod p^{\prime}$ и $s^{y}=h \bmod q^{\prime}$ относительно $x$ и $y$, используя алгоритм Полига-Хеллмана. Используя китайскую теорему об остатках, он может найти $e^{\prime}: e^{\prime}=x \bmod \left(p^{\prime}-1\right), e^{\prime}=y \bmod \left(q^{\prime}-1\right)$. Зная разложение $N^{\prime}$, он может найти $d^{\prime}=e^{\prime-1} \bmod \varphi\left(N^{\prime}\right)$, что завершает проведение атаки.

\section{3. Схема подписи Геннаро - Халеви - Рабина}

Данная схема подписи, являющаяся развитием схемы подписи RSA, была впервые предложена в [11]. Как и в случае RSA, заблаговременно вырабатывается набор доменных параметров $D$ : битовая длина простых сомножителей и хэш-функция hash, значения которой, будучи интерпретированы как целые числа, нечетные. Каждый участник вырабатывает ключевую пару, для чего выбирает два таких простых числа $p, q$, имеющих необходимую битовую длину, что $p \neq q$ и $(p-1) / 2,(q-1) / 2-$ простые, полагает $N=p q$ и выбирает случайное целое $t$. Открытым ключом является пара $(N, t)$, секретным - пара $(p, q)$. Алгоритм подписания $\Sigma_{G}$ при получении на вход кортежа $[M,(p, q), D]$, где $M$ - подписываемое сообщение, возвращает подпись $s=t^{h^{\prime}} \bmod N$, где $h^{\prime}$ определяется из уравнений $h^{\prime} h=1 \bmod (p-1)$ и $h^{\prime} h=1 \bmod (q-1)$, $h=\operatorname{hash}(M)$. Алгоритм $\Sigma_{V}$ проверки подписи на входе $[M, s, D,(N, t)]$ возвращает «Принять» тогда и только тогда, когда $s^{h} \bmod N=t$.

В той же работе [11] была показана стойкость схемы к экзистенциальной подделке на основе атаки с адаптивным выбором сообщений в модели случайного оракула при условии выполнения так называемого сильного предположения RSA, т. е. невозможности по данным $N=p q$, где $p, q$ неизвестны, и $s \in Z_{N}^{*}$ найти за время, ограниченное полиномом от битовой длины $N$, такую пару $(r, e)$, что $r^{e}=s \bmod N$. Однако в [16] показано, что данная схема является уязвимой к атаке DSKS (типа 1-ns-DSKS). Для этого противник просто выбирает новый случайный модуль $N^{\prime}$ и полагает $t^{\prime}=s^{h} \bmod N^{\prime}$. Очевидно, проверка подписи на открытом ключе $\left(N^{\prime}, t^{\prime}\right)$ пройдет успешно.

\section{4. Обобщенная схема подписи Эль-Гамаля}

Рассмотрим обобщенную схему подписи Эль-Гамаля. Заранее вырабатывается набор доменных параметров $D$ : циклическая группа $G, \# G=q$, где $q$ - простое число, $a$ - образующий элемент группы $G$, хэш-функция hash : $\{0,1\}^{*} \rightarrow\{0,1, \ldots, q-1\}$, отображение $R: G \backslash\left\{1_{G}\right\} \rightarrow\{0,1, \ldots, q-1\}$, где $1_{G}$ - единица группы $G$. 
Каждый участник вырабатывает свою ключевую пару $(d, y)$, в которой $d \in_{R}\{1,2, \ldots, q-1\}$ и $y=a^{d}$.

Подпись формируется следующим образом. Допустим, что необходимо подписать сообщение $M$. В ходе выполнения алгоритма подписи $\Sigma_{G}$ при входе $[M, d, D]$ выбирается секретное $k \in Z_{q}^{*}$, вычисляется $r=R\left(a^{k}\right)$, из уравнения $u=d v+k w(\bmod q)$, где $\{u, v, w\}=\{s, r, \operatorname{hash}(M)\}$, определяется значение $s$. Значение подписи полагается равным $(r, s)$.

Алгоритм проверки подписи $\Sigma_{V}$ на входе $[M,(r, s), D, y]$ возвращает «Принять» тогда и только тогда, когда верно следующее равенство:

$$
R\left(\left(a^{u} y^{-v}\right)^{w^{-1} \bmod q}\right)=r .
$$

Противник может провести успешную DSKS-атаку (типа 1- $d s$-DSKS), используя алгоритм, похожий на описанный в [16] для схемы подписи ECDSA, если он может подменить один из доменных параметров - образующий элемент группы $G$. Итак, предположим, что Алиса подписала сообщение $M$, используя обобщенную схему Эль-Гамаля, и получила значение подписи $(r, s)$. Противник желает получить другую ключевую пару $\left(d^{\prime}, y^{\prime}=a^{\prime d^{\prime}}\right)$, удовлетворяющую уравнению верификации, где $a^{\prime}-$ новый образующий элемент группы $G$. Противник получает уравнение

$$
R\left(\left(a^{u} y^{-v}\right)^{w^{-1} \bmod q}\right)=R\left(\left(a^{\prime u} y^{\prime-v}\right)^{w^{-1} \bmod q}\right) .
$$

Уравнение необходимо решить относительно $a^{\prime}$. Будем искать решение в виде $a^{\prime}=a^{t_{1}} y^{t_{2}}$. Перейдем к уравнению

$$
\begin{aligned}
& \left(a^{u} y^{-v}\right)^{w^{-1} \bmod q}=\left(a^{\prime u} y^{\prime-v}\right)^{w^{-1} \bmod q} \\
& \left(a^{u} y^{-v}\right)^{w^{-1} \bmod q}=\left(\left(a^{t_{1}} y^{t_{2}}\right)^{u}\left(\left(a^{t_{1}} y^{t_{2}}\right)^{d^{\prime}}\right)^{-v}\right)^{w^{-1} \bmod q .}
\end{aligned}
$$

Отсюда переходим к равенствам в показателях степеней и получаем систему относительно $\left(t_{1}, t_{2}\right)$ :

$$
\begin{aligned}
u & =t_{1} u-v d^{\prime} t_{1}, \\
-v & =t_{2} u-v d^{\prime} t_{2} .
\end{aligned}
$$

Получаем:

$$
\begin{aligned}
& t_{1}=u\left(u-v d^{\prime}\right)^{-1}, \\
& t_{2}=-v\left(u-v d^{\prime}\right)^{-1} .
\end{aligned}
$$


Таким образом, если противник выберет случайный секретный ключ $d^{\prime}$ : $d^{\prime} \neq u v^{-1} \bmod q$ и определит $a^{\prime}=a^{t_{1}} y^{t_{2}}=a^{u\left(u-v d^{\prime}\right)^{-1} \bmod q} y^{-v\left(u-v d^{\prime}\right)^{-1} \bmod q}$ и $y^{\prime}=a^{\prime d^{\prime}}$, то при проверке подписи с помощью открытого ключа $y^{\prime}$ получаем

$$
R\left(\left(\left(a^{\prime}\right)^{u}\left(y^{\prime}\right)^{-v}\right)^{w^{-1} \bmod q}\right)=R\left(\left(a^{u} y^{-v}\right)^{w^{-1} \bmod q}\right)=R\left(a^{k}\right)=r,
$$

т. е. равенство верификации выполняется. Это означает, что проверка подписи на ключевой паре, выработанной противником, прошла успешно. Получается, что при возможности изменения образующего элемента группы $G$ обобщенная схема Эль-Гамаля не является стойкой к атаке DSKS типа 1-ds-DSKS. Очевидно, при этом предположении не являются стойкими к данной атаке и частные случаи обобщенной схемы подписи Эль-Гамаля, в том числе определяемые стандартами электронной подписи России (ГОСТ Р 34.10-2012 [3]) и CША (ECDSA [10]), для которых были получены результаты о GMR-стойкости.

Так, для схемы подписи, определяемой стандартами ГОСТ Р 34.10-2001 и ГОСТ Р 34.10-2012, в так называемой модели с защищенным модулем (происходит подписание хэш-значения сообщения, зашифрованного доступным всем сторонам оракулом, содержащим ключ шифрования в секрете; это, очевидно, не затрудняет проведение атаки) в [1] было показано, что экзистенциальная подделка на основе атаки с адаптивным выбором сообщений не легче самой легкой из следующих задач: вычисления дискретного полулогарифма в используемой группе (т.е. нахождения в циклической группе $G$ с образующим элементом $g$ для случайного элемента $z \in G$ такой пары $(t, u)$, что $t=g^{u} z^{-t}$ ), нахождения коллизии используемой хэш-функции, проведения успешной IND-CPA атаки на используемый алгоритм шифрования. Для схемы ECDSA, в свою очередь, в [6] была показана стойкость к экзистенциальной подделке на основе атаки с адаптивным выбором сообщений в модели случайного оракула при условии трудноразрешимости задачи нахождения соответствующего дискретного полулогарифма.

Дополнительно можно показать, что для обобщенной схемы Эль-Гамаля можно построить и 2-коллизию по Роса с измененными доменными параметрами (т. е. провести атаку типа 2-ds-DSKS). Предположим, что необходимо, чтобы подпись $(r, s)$ проходила успешную верификацию как подпись сообщения $M^{\prime} \neq M$. Кортеж $\{u, v, w\}=\{s, r, \operatorname{hash}(M)\}$ при этом противником изменяется на $\left\{u^{\prime}, v^{\prime}, w^{\prime}\right\}=\left\{s, r, \operatorname{hash}\left(M^{\prime}\right)\right\}$. Таким образом, противник составит уравнение

$$
\left(a^{u} y^{-v}\right)^{w^{-1} \bmod q}=\left(\left(a^{t_{1}} y^{t_{2}}\right)^{u^{\prime}}\left(\left(a^{t_{1}} y^{t_{2}}\right)^{d^{\prime}}\right)^{-v^{\prime}}\right)^{w^{\prime-1} \bmod q},
$$


из которого следует, что

$$
\begin{aligned}
u w^{-1} & =\left(t_{1} u^{\prime}-v^{\prime} d^{\prime} t_{1}\right) w^{\prime-1}, \\
-v w^{-1} & =\left(t_{2} u^{\prime}-v^{\prime} d^{\prime} t_{2}\right) w^{\prime-1} .
\end{aligned}
$$

В итоге получаются выражения для $t_{1}, t_{2}$ :

$$
\begin{aligned}
& t_{1}=u w^{-1} w^{\prime}\left(u^{\prime}-v^{\prime} d^{\prime}\right)^{-1}, \\
& t_{2}=-v w^{-1} w^{\prime}\left(u^{\prime}-v^{\prime} d^{\prime}\right)^{-1} .
\end{aligned}
$$

Таким образом, противник может построить 2-коллизию по Роса для обобщенной схемы Эль-Гамаля за время, ограниченное полиномом от битовой длины ключевой пары.

Следует также отметить, что на схему электронной подписи ECDSA (а также на схемы электронной подписи, описываемые российским стандартом и основанные на использовании эллиптических кривых) может быть проведена атака типа 1-ns-DSKS, т. е. атака, не требующая подмены доменных параметров. Опишем ее (подробнее см. в [16]). Предположим, что легитимный владелец ключевой пары ECDSA желает провести атаку, описанную в разделе 2.2. Для этого он использует тот факт, что для $\operatorname{ECDSA} R(Q)=Q_{x}$, где $Q_{x}$ - абсцисса точки $Q$ используемой эллиптической кривой. Соответственно, для выполнения уравнения верификации подойдет как значение $V=w^{-1}(u P-v Y)=w^{-1}(u-v d) P$, так и $-V$, где $P-$ базовая точка, а $Y-$ открытый ключ законного владельца. Таким образом, если выработать новый секретный ключ по формуле

$$
d^{\prime}=v^{-1}(2 u-v d)
$$

то при проверке подписи с помощью открытого ключа $Q^{\prime}=d^{\prime} P$ равенство верификации будет выполнено, что завершает атаку. Далее такой тип атаки будем называть атакой с известным секретным ключом.

\section{5. Вариации схемы подписи Эль-Гамаля}

Те же рассуждения можно применить к схемам, которые являются дальнейшим обобщением схемы электронной подписи Эль-Гамаля, построены с использованием аналогичного математического аппарата, но используют вместо хэш-значения подписываемого сообщения хэш-код специального вида, а именно $h=\operatorname{hash}(f(M, r, q))$, где hash - криптографическая хэш-функция, $f$ - любая функция, для которой существует полиномиальная машина Тьюринга, вычисляющая ее правильно. Примером такой схемы является схема подписи Pointcheval-Vaudenay, описанная в [15], в которой используется $f(M, r, q)=M \| r$. 
Данная схема, очевидно, является уязвимой к атаке DSKS (типа 1- $d s$ DSKS), так как по сравнению со схемой Эль-Гамаля здесь лишь иначе рассчитывается хэш-код, а параметры функции $f$ в ходе атаки не подменяются. Необходимо отметить, что схема электронной подписи стандарта Республики Корея, вопрос уязвимости которой к атаке DSKS будет рассмотрен ниже, под определение обобщенной схемы подписи Эль-Гамаля со специальным хэшкодом не подходит, так как в данном случае хэш-значение рассчитывается с использованием параметров, которые в ходе атаки необходимо изменить.

Аналогично случаю обобщенной схемы Эль-Гамаля можно показать, что для схемы Pointcheval - Vaudenay также можно построить 2-коллизию по Роса, т. е. провести атаку типа 2-ds-DSKS.

\section{6. Схема Шнорра}

Рассмотрим теперь схему Шнорра ([26]). При использовании данной схемы заранее вырабатывается набор доменных параметров $D$, включающий такие простые числа $p, q$, что $q \mid p-1$, элемент $a$, для которого верно равенство $a^{q}=1 \bmod p$, и хэш-функцию hash $:\{0,1\}^{*} \rightarrow\{0,1, \ldots, q-1\}$. Каждый участник вырабатывает ключевую пару $(d, y)$; при этом секретный ключ $d$ определяется как $d \in_{R}\{1,2, \ldots, q-1\}$, а соответствующий открытый ключ рассчитывается по формуле $y=a^{-d}$.

Алгоритм подписания $\Sigma_{G}$ на входе $[M, d, D]$ возвращает $(e, s)$. Здесь $e=\operatorname{hash}(M \| r), r=a^{k}$, подпись $s$ определяется из уравнения $s=d e+k$, где $k \in \in_{R}\{1,2, \ldots, q-1\}$. Алгоритм $\Sigma_{V}$ проверки подписи на входе $[M,(e, s), D, y]$ возвращает сообщение «Принять» тогда и только тогда, когда $e=\operatorname{hash}(M \| z)$, где $z=a^{s} y^{e}$.

Для проведения успешной 1-ds-DSKS-атаки противник выбирает секретный ключ $d^{\prime} \neq d$. Затем ему необходимо подобрать параметр $a^{\prime}$ так, чтобы уравнение верификации выполнялось. Поскольку предполагается, что хэшфункция hash является стойкой к нахождению коллизий (collision-resistant), a также то, что сообщение не может быть изменено противником, для обеспечения равенства $\operatorname{hash}(M \| z)=\operatorname{hash}\left(M \| z^{\prime}\right)$ следует положить $z=z^{\prime}$. Запишем это равенство:

$$
a^{s} y^{e}=a^{\prime s} y^{\prime e}
$$

Представим $a^{\prime}$ в виде $a^{\prime}=a^{t_{1}} y^{t_{2}}$, где $t_{1}, t_{2} \in\{1,2, \ldots, q-1\}$, подставим это выражение в последующее равенство и теперь будем определять параметры $t_{1}, t_{2}$, удовлетворяющие равенству

$$
a^{s} y^{e}=a^{t_{1} s} y^{t_{2} s} a^{-d^{\prime} e t_{1}} y^{-d^{\prime} e t_{2}} .
$$


Переходим к равенствам в показателях степеней и получаем систему уравнений

$$
\begin{aligned}
& s=t_{1} s-d^{\prime} e t_{1}, \\
& e=t_{2} s-d^{\prime} e t_{2},
\end{aligned}
$$

откуда

$$
\begin{aligned}
& t_{1}=s\left(s-e d^{\prime}\right)^{-1}, \\
& t_{2}=e\left(s-e d^{\prime}\right)^{-1}
\end{aligned}
$$

при $d^{\prime} \neq e^{-1} s$.

Таким образом, если противник положит $a^{\prime}=a^{t_{1}} y^{t_{2}}$, где $t_{1}$ и $t_{2}$ удовлетворяют (14) и (15) соответственно, то верификация подписи $(e, s)$ на открытом ключе $y^{\prime}=a^{d^{\prime}}$ пройдет успешно, т. е. DSKS-атака (типа 1-ds-DSKS) завершится успехом.

Аналогично можно показать, что обобщенная схема электронной подписи Шнорра также не является стойкой к атаке типа 1-ds-DSKS.

Необходимо отметить, что задача построения 2-коллизии по Роса не проще задачи нахождения коллизии используемой в схеме хэш-функции. Честный подписывающий определяет параметр $e=\operatorname{hash}(M \| r)$, который является неизменяемой частью подписи. Если противник смог найти 2-коллизию по Роса, то это означает, что он сумел подобрать другое сообщение $M^{\prime} \neq M$ и $z^{\prime} \in\{1,2, \ldots, q-1\}$ так, что при проверке подписи будет выполнено равенство $e=\operatorname{hash}\left(M^{\prime}|| z^{\prime}\right)$, откуда следует

$$
\operatorname{hash}(M \| r)=\operatorname{hash}(M \| z)=\operatorname{hash}\left(M^{\prime} \| z^{\prime}\right),
$$

т. е. пара сообщений $M \| z$ и $M^{\prime} \| z^{\prime}$ является коллизией хэш-функции hash.

На схему электронной подписи Шнорра, основанную на использовании эллиптических кривых (см., например, [9]), как и на схему ECDSA, можно также провести атаку с известным секретным ключом без подмены доменных параметров (типа 1-ns-DSKS). Противник аналогично использует тот факт, что в качестве значения $z$ используется только абсцисса вычисляемой проверяющим точки кривой; если проверка подписи проводится для ключевой пары $\left(d^{\prime}, d^{\prime} P\right)$, где $d^{\prime}=-2 s e^{-1}-d$, а $P$ - базовая точка используемой группы точек эллиптической кривой, проверяющий получит обратную точку, при подстановке которой в уравнение верификации последнее выполнится. 


\section{7. Стандарт электронной подписи Республики Корея (KCDSA)}

Перейдем к рассмотрению схемы ЭП, описанной в стандарте Республики Корея ([27], далее - KCDSA). Вырабатывается набор доменных параметров $D$ : циклическая группа $G$ простого порядка $q$ с образующим элеменом $a$, хэш-функция $h:\{0,1\}^{*} \rightarrow\{0,1\}^{l}$, отображения $R_{1}: G \rightarrow\{0,1\}^{*}, R_{2}: G \rightarrow$ $\{0,1\}^{l}$, где $l-$ некоторый фиксированный параметр.

Каждый участник вырабатывает ключевую пару $(d, y)$, в составе которой $d \in \in_{R}\{1,2, \ldots, q-1\}-$ секретный ключ, а $y=a^{d^{-1} \bmod q}-$ открытый ключ.

Формирование подписи происходит следующим образом: алгоритм подписания $\Sigma_{G}$ на входе $[M, d, D]$ возвращает $(r, s)$, где $r=h\left(R_{1}\left(a^{k}\right)\right), s=$ $d\left(k-r \oplus h\left(R_{2}(y) \| M\right)\right)$, a $k \in_{R}\{1,2, \ldots, q-1\}$.

Алгоритм $\Sigma_{V}$ проверки подписи на входе $[M,(r, s), D, y]$ возвращает сообщение «Принять», если и только если

$$
h\left(R_{1}\left(y^{s} a^{r \oplus h\left(R_{2}(y) \| M\right)}\right)\right)=r .
$$

В [18] утверждается, что атаки на схему KCDSA, основанные на изменении параметров («parameter manipulation»), окончатся неудачей, так как при подписывании информация о циклической группе $G$ и ключе подписывающего добавляется к сообщению и хэшируется с ним. К сожалению, подробно это утверждение не поясняется. Однако атака, подобная описанной выше для схемы Шнорра или Эль-Гамаля, по-видимому, окончится неудачей в предположении стойкости хэш-функции $h$ к нахождению прообраза (preimage resistance) и второго прообраза (second preimage resistance). Предположим, что честный участник подписывает сообщение $M$ на ключевой паре $\left(d, y=a^{d^{-1}}\right)$, получает значение подписи $(r, s)$, а противник желает, изменив образующий элемент группы $a$ на $a^{\prime}=a^{t_{1}} y^{t_{2}}$, подобрать вторую ключевую пару $\left(d^{\prime}, y^{\prime}=a^{\prime d^{\prime}}\right)$, на которой верификация подписи $(r, s)$ пройдет успешно. Противнику требуется решить уравнение

$$
h\left(R_{1}\left(y^{s} a^{r \oplus h\left(R_{2}(y) \| M\right)}\right)\right)=h\left(R_{1}\left(y^{\prime s} a^{\prime r \oplus h\left(R_{2}\left(y^{\prime}\right) \| M\right)}\right)\right) .
$$

Ему либо придется искать второй прообраз хэш-функции, либо пытаться приравнять значения аргументов внешней хэш-функции, что приводит к системе уравнений

$$
\begin{array}{r}
s=t_{2} d^{\prime-1} s+t_{2}\left(r \oplus h\left(R_{2}\left(\left(a^{t_{1}} y^{t_{2}}\right)^{d^{\prime-1}}\right) \| M\right)\right), \\
r \oplus h\left(R_{2}(y) \| M\right)=t_{1} d^{\prime-1} s+t_{1}\left(r \oplus h\left(R_{2}\left(\left(a^{t_{1}} y^{t_{2}}\right)^{d^{\prime-1}}\right) \| M\right)\right)
\end{array}
$$

относительно $\left(t_{1}, t_{2}\right)$. 
Решение системы затруднено тем, что в ее уравнения входят значения хэшфункции от выражения, содержащего неизвестные.

Рассмотрим ситуацию, когда атаку проводит нечестный подписывающий. Итак, пусть сообщение подписано им на ключевой паре $\left(d, y=a^{d^{-1}}\right)$, где $d=1$. Тогда, сложив уравнения системы (16), (17), получим уравнение относительно $\left(t_{1}+t_{2}\right)$ :

$$
\begin{aligned}
& s+r \oplus h\left(R_{2}(y) \| M\right)= \\
& =\left(t_{2}+t_{1}\right)\left(d^{\prime-1} s+r \oplus h\left(R_{2}\left(a^{\left(t_{1}+t_{2}\right) d^{\prime-1}}\right) \| M\right)\right) .
\end{aligned}
$$

Переписываем его в виде

$$
\begin{aligned}
& h\left(R_{2}\left(a^{\left(t_{1}+t_{2}\right) d^{\prime-1}}\right) \| M\right)= \\
& =\left(\left(s+r \oplus\left(h\left(R_{2}(y) \| M\right)\right)\right)\left(t_{2}+t_{1}\right)^{-1}-d^{\prime-1} s\right) \oplus r .
\end{aligned}
$$

Если при некотором фиксированном $d^{\prime}$ противник может найти решение системы, а следовательно, и уравнения, то он получит решение уравнения вида

$$
h(f(t))=g(t),
$$

где $t=t_{1}+t_{2}$, а $g(t)$ - обратимая функция. Для того чтобы правая часть уравнения не зависела от $t$, надо обратить в 0 коэффициент перед $\left(t_{2}+t_{1}\right)^{-1}$. Этот коэффициент равен $s+r \oplus\left(h\left(R_{2}(y) \| M\right)\right)$. Нечестный подписывающий мог при подписывании взять $k$ равным 0 . Так как по предположению $d=1$, TO

$$
s=-\left(r \oplus\left(h\left(R_{2}(y) \| M\right)\right),\right.
$$

т.е. коэффициент обнуляется. Таким образом, если бы противник мог решить систему, то он мог бы решить уравнение $h(f(t))=$ const относительно переменной $t, \quad$ т. е. найти прообраз значения хэшфункции:

$$
h^{-1}(\text { const })=f(t) .
$$

Тем самым доказано следующее утверждение.

Утверждение 1. Задача поиска коэффициентов перехода к новому образующему элементу используемой группы схемы KCDSA для проведения успешной DSKS-атаки не легче задачи нахождения прообраза и задачи нахождения второго прообраза используемой в схеме подписи хэщ-функции. 


\section{8. Схема Крамера-Шоупа}

В [7] Крамер и Шоуп предложили схему, основанную на задаче факторизации большого целого числа, для которой удалось показать стойкость к экзистенциальной подделке на основе атаки с адаптивным выбором сообщений. Для использования схемы заранее вырабатывается набор доменных параметров $D$ : битовые длины используемых простых чисел и тип используемой хэш-функции. Подписывающий вырабатывает ключевую пару следующим образом: выбирает простые числа $p, q$ необходимой битовой длины, определяет $n=p q$, выбирает квадратичные вычеты $x, h$ по модулю $n$, случайное простое число $e^{\prime}$ заданной битовой длины. Открытый ключ - кортеж $\left(n, h, x, e^{\prime}\right)$, секретный ключ - $(p, q)$. Формирование подписи сообщения $M$ происходит следующим образом: в ходе выполнения алгоритма подписания $\Sigma_{G}$ на входе $[M,(p, q), D]$ генерируются простое $e \neq e^{\prime}$ той же битовой длины и произвольный квадратичный вычет $y^{\prime}$. Далее из уравнения $y^{\prime e^{\prime}}=x^{\prime} h^{\text {hash }(M)} \bmod n$ определяется значение $x^{\prime}$, а из уравнения $y^{e}=x h^{\operatorname{hash}\left(x^{\prime}\right)} \bmod n$ получается $y$ (зная разложение $n$, подписывающий может сделать это легко). Подписью является кортеж $\left(e, y, y^{\prime}\right)$. Проверка подписи происходит следующим образом: проверяется, что $e-$ нечетное число заданной битовой длины, вычисляется $x^{\prime}=\left(y^{\prime}\right)^{e^{\prime}} h^{-\operatorname{hash}(M)} \bmod n$. Алгоритм проверки подписи возвращает «Принять» тогда и только тогда, когда равенство $x=y^{e} h^{-\operatorname{hash}\left(x^{\prime}\right)} \bmod n$ верно.

В [7] для данной схемы было доказано следующее утверждение.

Утверждение 2. Описанная схема является стойкой к экзистенциальной подделке подписи на основе атаки с адаптивным выбором сообщений, если используемая хэш-функиия является стойкой к нахождению коллизий и выполнено усиленное предположение RSA, m. е. по данному целому п $u z \in Z_{n}^{*}$ «трудно» найти такие $r>1$ и $y \in Z_{n}^{*}$, что $y^{r}=z$.

Однако данная схема уязвима к атаке типа 1-ns-DSKS. Предположим, что противник обладает сообщением $m$ и подписью $\left(e, y, y^{\prime}\right)$. Тогда он выбирает такие новые параметры $\bar{p}$ и $\bar{q}$, что $y^{\prime}-$ квадратичный вычет по модулю $\bar{n}=\bar{p} \cdot \bar{q}$ и $y<\bar{n}, y^{\prime}<\bar{n}$. Затем он случайным образом выбирает параметры $\bar{h}, \overline{e^{\prime}}$ так, что $\bar{h}-$ квадратичный вычет по модулю $\bar{n}$, а $\overline{e^{\prime}} \neq e$ простое. Далее из уравнения $y^{\overline{e^{\prime}}}=\overline{x^{\prime}} \bar{h}^{\operatorname{hash}(m)}$ он получает $\overline{x^{\prime}}$, а затем из уравнения $y^{e}=\bar{x} \bar{h}^{\text {hash }\left(\overline{x^{\prime}}\right)}$ получает $\bar{x}$. Если $\bar{x}-$ невычет, то атака начинается заново, в противном случае противник заявляет открытый ключ $\left(\bar{n}, \bar{h}, \bar{x}, \overline{e^{\prime}}\right)$. Так как разложение $\bar{n}$ ему известно, он сможет получить действительный сертификат ключевой пары, проверка подписи на которой пройдет успешно по построению. 


\section{9. Схема Лампорта}

Схема Лампорта предложена в [17]. Для наглядности опишем эту схему, следуя [4, стр. 93].

Предположим, что существует однонаправленная функция

$$
f: K \rightarrow V, \quad \text { где } K \text { и } V-\text { произвольные множества }
$$

(в [4] вместо однонаправленной функции рассматривается симметричное шифрование). Множества $K, V$ и функция $f$ суть доменные параметры схемы. Подписывающий для подписания $n$-битного сообщения $M=m_{1} \ldots m_{n}$ вырабатывает $n$ пар

$$
\left(k_{i, 0}, k_{i, 1}\right) \in K \times K, \quad k_{i, 0} \neq k_{i, 1},
$$

затем он определяет $v_{i, j}$ :

$$
v_{i, j}=f\left(k_{i, j}\right), \quad i=1,2, \ldots, n, j=0,1 .
$$

Значения $v_{i, j}$ формируют открытый ключ, а $k_{i, j}$ - секретный. Подпись сообщения $M$ формируется так: $s=\left(k_{1, m_{1}}, k_{2, m_{2}}, \ldots, k_{n, m_{n}}\right)$. Для верификации проверяются равенства

$$
f\left(k_{i, m_{i}}\right)=v_{i, m_{i}} \quad \text { для всех } \quad i \in\{1,2, \ldots, n\} .
$$

Необходимо отметить, что подпись одноразовая, так как при подписании вскрывается часть секретного ключа.

Схема, очевидно, является уязвимой к атаке типа 1-ns-DSKS. Противник, зная $M=m_{1} m_{2} \cdots m_{n}$ и значение подписи $\left(k_{1, m_{1}}, k_{2, m_{2}}, \ldots, k_{n, m_{n}}\right)$, может случайным образом сгенерировать остальные секретные ключи $k_{i, \overline{m_{i}}}^{\prime}$, получить для них открытые ключи $z_{i, \overline{m_{i}}}^{\prime}=f\left(k_{i, m_{i}}^{\prime}\right)$, а $z_{i, m_{i}}^{\prime}=z_{i, m_{i}}$. Очевидно, что на таком открытом ключе верификация подписи также пройдет корректно. Атака может быть расширена и на многоразовый вариант схемы, описанный Наором и Юнгом в [20], в котором используются семейства универсальных однонаправленных хэш-функций. Противник точно таким же образом дополняет свой секретный ключ случайными значениями, создает новый лист-состояние, подписывает его при помощи имеющегося у него текущего листа. В результате проверяющий может, верифицируя цепочку подписей, дойти до доверенного листа злоумышленника и убедиться в том, что подписывание производил именно он. В той же работе показана стойкость данной схемы к экзистенциальной подделке на основе атаки с адаптивным выбором сообщений в предположении существования однонаправленной перестановки. 


\section{4. Противодействие атаке DSKS}

В этом разделе будут рассмотрены меры, которые можно применять для предотвращения проведения атаки DSKS на схемы электронной подписи.

\section{1. Затруднение подмены параметров}

Как показано выше, атака DSKS на схемы Эль-Гамаля и Шнорра становится возможной, если противник может подменять доменные параметры. Помешать ему можно несколькими способами.

Первый - это прямой запрет на изменение доменного параметра (например, образующего элемента в случае схемы Эль-Гамаля). В частности, в национальном стандарте Республики Беларусь ([2]) указывается, что образующий элемент задается однозначно параметрами эллиптической кривой и не может быть изменен.

К сожалению, этот метод защиты не спасает, например, от варианта атаки с известным секретным ключом на варианты схем Эль-Гамаля и Шнорра, основанные на использовании эллиптических кривых. Связано это с тем, что в уравнение верификации входит значение только одной координаты точки эллиптической кривой, а не обеих, т. е. появляется возможность найти другую точку, которая тоже будет удовлетворять условию проверки.

Это подсказывает второй способ улучшения схем электронной подписи: необходимо, чтобы компоненты алгебраических объектов, используемые в уравнении верификации, описывали данный объект однозначно.

\section{2. Особый порядок генерации параметров}

Для повышения сложности атаки DSKS в стандартах схем электронной подписи можно включать способы генерации ключевых пар и открытых параметров из случайных значений seed. Основная идея, сформулированная Томашом Роса в [23], заключается в том, что никто, даже законный владелец ключевой пары, не должен иметь возможности генерировать ключевую пару, которая совпадет с заранее выбранным значением.

В [10] для генерации секретного ключа, одноразового параметра безопасности и образующего элемента циклической группы используются функции, для которых неизвестны эффективные алгоритмы нахождения прообраза. Честный подписывающий имеет эти значения и может в случае необходимости предъявить их арбитру, а противнику придется инвертировать однонаправленную функцию, чтобы предъявить случайное значение seed', по которому он якобы получил данные параметры. Хэш-значения случайных битовых последовательностей, по которым генерируется долговременная ключевая информация, можно добавлять в сертификат открытого ключа. 
Также имеет смысл проверять, имеют ли открытые и секретные параметры заявленные свойства. Например, в случае атаки на схему RSA, которая основана на том, что противник подбирает простые $p$ и $q$ так, что $p-1$ и $q-1$ раскладываются на малые множители, арбитр при предъявлении секретного ключа может проверить, являются ли $p$ и $q$ безопасными простыми числами, и в том случае, когда это не так, утверждать, что ключевая пара создана для атаки.

\section{3. Использование специального формата сообщения}

Как правило, в схемах электронной подписи подписывается не само сообщение, а его хэш-код, для получения которого используется криптографическая хэш-функция. Для затруднения проведения атаки DSKS можно подписывать не только само сообщение, но и дополнительную информацию, такую, например, как доменные параметры или открытый ключ (как в схеме KCDSA). При попытке атаки на схему ЭП, которая устроена таким образом, противник столкнется с необходимостью решать уравнение, в которое будет входить значение хэш-функции от доменных параметров и которое надо разрешить относительно доменных параметров, что, по-видимому, сложно.

Также можно внедрять в подписываемое сообщение метки времени, указания на адресата и другие реквизиты, но необходимо иметь в виду, что при наличии возможности построения 2-коллизии по Роса для используемой схемы электронной подписи данные меры неэффективны, так как противник может видоизменять сообщение.

\section{4. Защита протоколов}

Как было указано выше, возможность проведения атаки DSKS на схемы электронной подписи делает уязвимыми некоторые криптографические протоколы, использующие данные схемы как примитив. Описанные выше способы позщволяют с этим бороться.

Например, противодействовать атаке на протокол выработки общего ключа STS, описанной в разделе 2.3, можно способом, предложенным в [5], где рассматривается протокол ISO-STS-MAC (ISO/IEC 11770-3 Key agreement mechanism 7), описанный в [14]:

1) $A \rightarrow B: a^{x}, \operatorname{cert}_{A}$;

2) $B \rightarrow A: a^{y}, s_{B}\left(a^{x}\left\|a^{y}\right\| \operatorname{id}_{B}\right), \operatorname{MAC}_{K}\left(s_{B}\left(a^{x}\left\|a^{y}\right\| \operatorname{id}_{B}\right)\right), \operatorname{cert}_{B}$;

3) $A \rightarrow B: s_{A}\left(a^{x}\left\|a^{y}\right\| \operatorname{id}_{A}\right), \operatorname{MAC}_{K}\left(s_{A}\left(a^{x}\left\|a^{y}\right\| \operatorname{id}_{A}\right)\right)$. 
Такая модификация протокола делает невозможным проведение атаки типа unknown key share (UKS) описанным в разделе 2.3 способом, так как противник, который может провести DSKS-атаку, сможет только подобрать новую ключевую пару, на которой верификация подписи $s_{A}\left(a^{x}\left\|a^{y}\right\| \mathrm{id}_{A}\right)$ будет проходить успешно, но участник $B$, будучи уверенным, что сообщение пришло от $M$, проверит полученную подпись как подпись сообщения $s_{M}\left(a^{x}\left\|a^{y}\right\| \operatorname{id}_{M}\right)$ и выявит несоответствие. Подменить же подпись противник из-за наличия МАC-значения не сможет.

Следует заметить, что если можно построить 2-коллизию по Роса для данной схемы электронной подписи, то такое улучшение не поможет, так как противник $M$ сможет найти ключевую пару, для которой

$$
s_{M}\left(a^{x}\left\|a^{y}\right\| \operatorname{id}_{M}\right)=s_{A}\left(a^{x}\left\|a^{y}\right\| \operatorname{id}_{A}\right),
$$

и довести атаку UKS до конца. От этого можно защититься, подписывая не только идентификатор абонента, но и его открытый ключ или сертификат целиком, либо внести данный сертификат в состав сообщения, от которого вычисляется МАС-значение.

Другим способом, причем универсальным, защиты произвольного криптографического протокола от атак, связанных с атакой DSKS на используемый примитив электронной подписи, является требование подписания не менее двух сообщений на той же ключевой паре в рамках выполнения сеанса протокола. Противнику в таком случае потребуется подбирать ключи так, чтобы подпись совпадала на двух и более сообщениях, а не на одном, что выходит за рамки определения атаки DSKS, а вопрос о возможности подбора такой ключевой пары пока открыт.

Однако можно показать (например, для схемы Эль-Гамаля), что в ходе решения задачи о поиске коэффициентов перехода к новому образующему элементу можно получить равенства (обозначения те же, что и в разделе 3.4 )

$$
\begin{gathered}
u_{1}\left(u_{1}-v_{1} d^{\prime}\right)=t_{1}=u_{2}\left(u_{2}-v_{2} d^{\prime}\right), \\
-v_{1}\left(u_{1}-v_{1} d^{\prime}\right)=t_{2}=-v_{2}\left(u_{2}-v_{2} d^{\prime}\right) .
\end{gathered}
$$

Таким образом, получена система двух линейных уравнений относительно одной неизвестной $d^{\prime}$, которая, учитывая случайность коэффициентов, в общем случае совместной не является. 
Необходимо отметить, что если противник все же может для некоторой схемы электронной подписи подбирать такие ключевые пары, данный способ затруднения атаки на протокол все равно будет эффективным, если атакуемый протокол подразумевает интерактивный обмен сообщениями, так как противнику придется получить данную ключевую пару (и, возможно, сертификат открытого ключа) до того, как он получит второе значение подписи честного участника.

В качестве примера, где такой прием успешно работает, можно рассмотреть описанную в [4, с. 141] модификацию протокола сертифицированной почты, атака на который была предложена в разделе 2.4. Он защищен от этой атаки, так как адресат должен переслать отправителю, помимо $s_{B}(M)$, еще и

$$
s_{B}(K)=\Sigma_{G}\left(K, \operatorname{priv}_{B}, D\right),
$$

что вынуждает его подбирать ключевую пару так, чтобы верификация проходила успешно сразу на двух сообщениях.

Еще одним важным способом предотвращения атаки DSKS являются метки времени (timestamp), которые создаются третьей доверенной стороной и правильность которых можно проверить.

Если честный участник ставит подпись под сообщением, на котором есть метка времени, то дата выдачи сертификата открытого ключа, полученного противником, будет позже даты, указаной в метке, что выдаст факт проведения атаки. Если вновь вернуться к протоколу сертифицированной почты, то при использовании меток времени отправитель сможет заметить, что сертификат открытого ключа противника выдан после того, как он отправил письмо, что явно укажет на факт проведения атаки.

\section{5. Выводы}

1. Продемонстрировано, что уязвимость схемы электронной подписи к атаке дублирования подписи на выбранном ключе может повлечь за собой уязвимость протоколов, использующих данную схему в качестве примитива, к некоторым атакам. Построен пример такой атаки на протокол сертифицированной почты Шнайера-Риордана. Примером подобного рода атак также является атака Uknown Key Share на протокол выработки общего ключа STS, описанная в [5]. 
2. Предложены практически реализуемые атаки дублирования подписи на выбранном ключе на обобщенные схемы электронной подписи Эль-Гамаля и Шнорра в случае, когда противник имеет возможность изменять образующий элемент используемой группы. Одновременно описан способ построения 2коллизий по Роса для схемы Эль-Гамаля, наличие которых может создать дополнительные возможности для атак на прикладные протоколы, как это было показано для протокола ISO-STS-MAC. Доказано, что для схемы Шнорра построение 2-коллизии по Роса не проще решения задачи построения коллизии используемой хэш-функции.

3. Доказано, что задача нахождения нового образующего элемента, используемого в атаках типа 1-ds-DSKS на схемы Эль-Гамаля и Шнорра, для схемы электронной подписи KCDSA полиномиально сводится к задаче построения прообраза или второго прообраза используемой хэш-функции.

4. Показано, что схемы Крамера - Шоупа и Лампорта, для которых получены оценки стойкости к экзистенциальной подделке на основе атаки с адаптивным выбором сообщений, являются уязвимыми по отношению к атаке дублирования подписи на выбранном ключе.

Таким образом, большинство современных схем электронной подписи являются уязвимыми по отношению к атаке DSKS. Уязвимость схем электронной подписи к атаке DSKS не означает, что они являются нестойкими, но этот факт должен учитываться при использовании данных схем в качестве примитивов в более сложных криптографических механизмах.

Защита от атаки дублирования подписи на выбранном ключе достигается без ущерба практической эффективности путем применения таких широко распространенных мер, как:

- обязательное использование специальных алгоритмов выбора доменных параметров и ключей,

- запрет подмены доменных параметров схемы подписи,

- включение в сообщение данных сертификата открытого ключа и реквизитов сообщения,

- использование меток времени,

а также следующих мер, предложенных в данной работе:

- выбор такого уравнения верификации при синтезе схемы электронной подписи, при котором компоненты алгебраических структур, используемых в данном уравнении, описывают данные структуры однозначно,

- подписывание как минимум двух различных сообщений с использованием одной ключевой пары. 


\section{Список литературы}

[1] Варновский Н. П., “Стойкость схем электронной подписи в модели с защищенным модулем”, Дискретная математика, $20: 3$ (2008), 147-159.

[2] Государственный стандарт Республики Беларусь «Информационные технологии и безопасность. Алгоритмы электронной цифровой подписи и транспорта ключа на основе эллиптических кривых》 (СТБ 34.101.45-2013), Утвержден и введен в действие постановлением Госстандарта Республики Беларусь от 30 августа 2013 г. № 45, Минск: Госстандарт, 2013, 40 c.

[3] Национальный стандарт Российской Федераџии «Информаџионная технология. Криптографическая защита информации. Прочессы формирования и проверки электронной ицифровой подписи» (ГОСТ Р 34.10-2012), Утвержден и введен в действие П риказом Федерального агентства по техническому регулированию и метрологии от 7 августа 2012 г. № 215-ст, М.: Стандартинформ, 2012.

[4] Черемушкин А.В., Криптографические протоколь. Основные свойства и уязвимости, М.: Изд. центр «Академия», 2009, 272 с.

[5] Blake-Wilson S., Menezes A., "Unknown key-share attacks on the station-to-station (STS) protocol”, Lect. Notes Comput. Sci., 1560 (1999), 156-170.

[6] Brown D., "On the Provable Security of ECDSA". In: "Advances in Elliptic Curve Cryptography" /под общ. ред. I. F. Blake, G. Seroussi, N. P. Smart, Cambridge: Cambridge Univ. Press, 2005, 21-40.

[7] Cramer R., Shoup V., "Signature schemes based on the strong RSA assumption", ACM Trans. Inform. System Security, 3:3 (2000), 161-185.

[8] Diffie W., van Oorschot P. C., Wiener M. J., "Authentication and authenticated key exchanges", Designs, Codes and Cryptography, 2 :2 (1992), 107-125.

[9] Elliptic Curve Cryptography, version 2.0, Technical Guideline TR-03111, Bonn: Bundesamt fuer Sicherheit in der Informationstechnik, 2012

[10] FIPS PUB 186-4: Digital Signature Standard (DSS), Gaithersburg, MD: National Institute of Standards and Technology, 2013, iv+121 c., http://nvlpubs.nist.gov/nistpubs/FIPS/ NIST.FIPS.186-4.pdf.

[11] Gennaro R., Halevi S., Rabin T., "Secure hash-and-sign signatures without the random oracle". In: "Advances in Cryptology - EUROCRYPT'99", International Conference on the Theory and Application of Cryptographic Techniques. (Prague, May 2-6, 1999). Proceedings, Lect. Notes Comput. Sci., 1592 /под общ. ред. J. Stern, Heidelberg etc.: Springer, 1999, 123-139., http://dblp.uni-trier.de/rec/bib/conf/eurocrypt/99.

[12] Goldwasser S., Micali S., Rivest R.L., "A digital signature scheme secure against adaptive chosen-message attacks", SIAM J. Comput., 17:2 (1988), 281-308.

[13] GoldwasserS., MicaliS., RivestR.L., "A "paradoxical" solution to the signature problem". In: "Proceedings of the 25th Annual Symposium on Foundations of Computer Science", (Singer Island, Fla., October 24-26, 1984) /под общ. ред. L. Valiant, L. Stockmeyer, R.. Karp, M. O'Donnell, L. Gubias, and M. Tompa, Piscataway, NJ: IEEE / Computer Society Press, 1984, 441-448.

[14] ISO/IEC 11770-3 Information technology - Security techniques - Key management - Part 3: Mechanisms using asymmetric techinques. 
[15] ISO/IEC 14888-3 Information technology - Security techniques - Digital signatures with appendix - Part 3: Discrete logarithm based mechanisms.

[16] Koblitz N., Menezes A., "Another look at security definitions", IACR Cryptology ePrint Archive, № Report 2011/343, 39 c., http://eprint.iacr.org/2011/343.

[17] Lamport L., Constructing Digital Signatures from a One Way Function, Menlo Park, CA: SRI International/Computer Sci. Lab., 1979, 7 c.

[18] Lee P. J., Lim C.H., "A Study on the proposed Korean Digital Signature Algorithm”. In: " $A d$ vances in Cryptology - ASIACRYPT'98", International Conference on the Theory and Application of Cryptology and Information Security (Beijing, China, October 18-22, 1998). Proceedings, Lect. Notes Comput. Sci., 1514 /под общ. ред. K. Ohta, D. Pei, Heidelberg etc.: Springer, 1998, 175-186.

[19] Menezes A., Smart N., "Security of signature schemes in a multi-user setting", Designs, Codes and Cryptography, $33: 3$ (2004), 261-274.

[20] Naor M., Yung M., "Universal one-way hash functions and their cryptographic applications". In: "Proceedings of the 21st Annual ACM Symposium on Theory of Computing" / под общ. ред. D. S. Johnson, N. Y.: Ass. Comput. Machinery, 1989, 33-43.

[21] Rivest R. L., Shamir A., Adleman L., "A method for obtaining digital signatures and public-key cryptosystems", Comm. ACM, $21: 2$ (1978), 120-126.

[22] Rosa T., "Key-collisions in (EC)DSA schemes: Attacking non-repudiation", A talk presented at rump session of 22nd Annual International Cryptology Conference (Santa Barbara, CA, August 18-22, 2002)., IACR Cryptology ePrint Archive, № Report 2002/129 (2002), 13 c., http://eprint.iacr.org/2002/129.

[23] Rosa T., "On the key-collisions in the signature schemes". In: "Proceedings of the Czech Cryptologic Workshop VKB 2002", (Brno, April 3-4, 2002), 2002, 14-26., http://crypto.hyperlink.cz/files/k-kolize_rosa.pdf (на чешском яз.).

[24] Schneier B., Riordan J., "A certified e-mail protocol". In: "Proceedings of the 14th Annual Computer Security Applications Conference", (Phoenix-Scottsdale, AZ, December 7-11, 1998), Los Alamitos, CA, etc.: IEEE Computer Society, 1999, 347-352.

[25] Schneier B., Hall C., "An improved e-mail protocol". In: "Proceedings of the 13th Annual Computer Security Applications Conference", (San Diego, CA, December 8-12, 1997), Los Alamitos, CA, etc.: IEEE Computer Society, 1997, 227-230.

[26] Schnorr C. P., "Efficient signature generation by smart cards", J. Cryptology, 4:3 (1991), 161174 ..

[27] TTAK.KO-12 / R2. Digital signature mechanism with appendix - Part 2 - Certificate-based digital signature algorithm. 